\title{
Developing a program for pediatric urological care in the community
}

\author{
Frank Papanikolaou, MD;' Linda Lee, $M D^{2}$
}

'University of Toronto, The Hospital for Sick Children, and Trillium Health Partners, Toronto, ON, Canada; 2Division of Urology, Department of Surgery, Vancouver Island Health Authority, Victoria, BC, Canada

Cite as: Can Urol Assoc J 2017;11(1-2Suppl1):S93-5. hitp://dx.doi.org/10.5489/cuaj.4335

See related commentary on page $\$ 96$.

\section{Introduction}

The growth of large metropolitan areas across Canada has fostered the need to provide tertiary care to patients outside of the traditional university-affiliated hospitals. Subspecialty urology care at centres of excellence in the community includes urological oncology, men's health, community urology, and pediatric urology. The two of us have developed such centres of excellence in pediatric urology in the community settings of Mississauga, $\mathrm{ON}$, and Victoria, BC. This article highlights personal experiences in developing these programs and the lessons learned. It is hoped that this can help guide similar undertakings by others to develop centres of excellence in subspecialty urology care so as to bring care closer to patients' homes.

\section{Dr. Papanikolaou's perspective}

Mississauga is a city with a population of over 700000 . The Credit Valley Hospital serves not only Mississauga, but a surrounding catchment area of Brampton and Oakville and is nestled between McMaster University and the University of Toronto. When patients and parents in the catchment area are given the opportunity to have care provided closer to home, many choose to stay close to home.

In this context, I have been fortunate to have had the support to develop a program of tertiary pediatric urological care in a community setting at the Credit Valley Hospital, outside of a university-affiliated hospital. Subsequent to instituting this program, the Credit Valley Hospital has become affiliated with the University of Toronto and this has facilitated a greater collaboration between the community and the academic centre.

With the training I received in a two-year pediatric urology fellowship at the Hospital for Sick Children, and with the support of my practice group and the institution, I was able to confidently develop the program at the Credit Valley Hospital. There were challenges to overcome in developing the program. Although some patients and parents prefer the medical care to be provided closer to home, others prefer the allure of the university hospital. However, the large population of patients and relative lack of participation by other local urologists regionally to perform pediatric procedures, generated a sufficient volume of care for me to become the designated pediatric urologist in the region.

My vision was to create an outstanding program to provide care close to home. It was both with purposeful strategy and serendipity that I came across the keys to developing the pediatric urology program at the Credit Valley Hospital. Creating excitement and buy-in from my colleagues and the administration to prioritize pediatric urology proved to be the foundation for further growth of the program. Furthermore, the more people began to believe in the idea, the more the idea came to life.

There were, of course, challenges faced in developing the program and this provided an impetus for me to acquire more leadership skills through formal studies at the Rotman School of Management and the Schulich School of Business at York University. These programs and the leadership development I received formalized the processes that helped me to promote the program.

Developing the program necessitated a number of requisites be filled. The program needed physical resource in the clinic and operating room, as well as surgical equipment. We also needed to build the team, grow a practice with referrals, and then maintain a program of quality and excellence. We lobbied hard to create a weekly, dedicated pediatric urology clinic through countless meetings and roadblocks. Having a dedicated pediatric urology clinic put patients and parents at ease that they were being cared for in an environment conducive to pediatric care. We were successful with perseverance.

It was with the support of my division chief that it was possible to make the capital investment to purchase the equipment to have a viable pediatric surgical practice. This included pediatric rigid and flexible cystoscopies, microsurgical instruments, and pediatric laparoscopic equipment. 
The postoperative management of the pediatric patient was improved by a segregated recovery room area for pediatric patients and parents.

The most critical part of the journey was not acquiring the physical space and equipment, but rather developing the team. It was not an easy process to build the team and it has taken years to reach the potential that I was hoping for. The journey reaffirmed for me that healthcare is a team sport. As surgeons, however, we give very individualized care. We operate and focus on improving our own surgical outcomes. However, our patients see their care as provided by all the people who come into contact with them during their visit. It was clear that the team had to be a part of the vision for the program. I set out to educate our clinic, ward, operative, and recovery room nurses. I also empowered them to teach others. I recruited an early childhood educator to do arts and crafts in the clinic. I helped to create a program with a child support worker to teach kids about what will happen to them on the day of surgery. The department of diagnostic imaging needed to be part of the team. The chief of radiology and I standardized the initially poor subjective reporting of ultrasound reports for the grading of hydronephrosis. We set up radiology rounds with the pediatricians to better understand each other's clinical decision-making. I also developed a strong rapport with department of diagnostic imaging at SickKids so that I could review complex cases. The pediatricians at Credit Valley have always been supportive with their referrals and with postoperative care comanagement.

Amongst the successes, unfortunately, there are obstacles, and not everyone was supportive of the program. Initially, there was resistance by some members of the anesthesia department to undertake anesthesia for longer cases and laparoscopic surgery. I initially took it upon myself to convince the individuals to reconsider their reluctance. This approach, unfortunately, didn't work. Ultimately, I organized an academic rounds session with a visiting professor from SickKids and this allayed the concerns of the anesthetists. I then went on introduce pediatric laparoscopy at Credit Valley.

Concomitantly, as I built the program, I also built up my practice. One of the unexpected challenges I faced was organizing talks for the family physicians and pediatricians. It was a challenge to get a sponsor for a pediatric urology talk. One of the solutions proved to be to give a talk on an adult urology topic and then a presentation on pediatric urology. I also fostered a network of referrals from my colleagues in urology.

After successfully building my practice and the program, we decided it was time to scale up. The chief of surgery and I created a division of pediatric surgery, which I lead. We have gone on to expand the volume and range of services provided to pediatric patients.
In order to maintain excellence, especially as a solo pediatric urologist, I undertook a lot of continuing medical education. I took courses at the American Academy of Pediatrics and surgical courses in the U.S. I worked in Africa with a senior pediatric urologist doing complex reconstructive cases. I participated as a European Urological Association visiting fellow for one month in urological centres in Europe. I invited visiting pediatric urologists from other centres to visit and assist me with complex reconstructive cases. I sought the advice of my colleagues at Sick Kids when needed. Our team is further challenging itself by now sharing these skills in the Credit Valley urology fellowship program we developed.

I believe that creating meaningful connections and community is part of what our healthcare system requires. Credit Valley Hospital and Sick Kids pediatric urology are working together in this regard to improve access to care.

\section{Dr. Lee's perspective}

Pediatric urology expertise is often thought to be a subspecialty isolated to major academic centres. This may be a product of our training as residents, as we acquired our pediatric urology experience at these centres. A pediatric urologist in the community is less commonplace. However, there are key advantages to establishing such practices as well.

The most compelling argument for community pediatric urology is the delivery of care closer to home. For parents, it means decreased travel expenses and less time off work. For patients, it means less time off school and timelier access to specialized care. At the institutional level, it not only allows for specialized level of care to be delivered, but also expands what other departments are able to offer (e.g. neonatal intensive care unit [NICU], pediatric intensive care unit [PICU], pediatric surgery, pediatric gynecology, etc.). Pediatric urology patients can be funneled to the dedicated pediatric urologist, allowing adult urology colleagues to focus on their own practices.

For the new pediatric urologist graduating from fellowship, there are reasons to consider a community practice as well. Some may enjoy the clinical aspects without the pressure of academic obligations (e.g., publication, teaching, etc.). Meanwhile, a community practice does not preclude these academic activities for those who seek them out. By keeping an open mind to community practice, one is not restricted to major academic centres and this may translate to more job opportunities. For some, it may also mean flexibility in their workload that they may not have in an academic position.

Typically, academic pediatric urologists are funded through an Alternate Funding Plan (AFP). Most community pediatric urologists are paid based on a fee-for-service model 
and see a combination of both pediatric and adult patients. Financial reasons and the pediatric urology fee schedule are often cited as reasons for this. In my opinion, it is also great to be able to keep one's adult urology skills. A pediatric urologist's skill set is surprisingly broad. In fellowship, we performed cases in endourology, minimally invasive surgery, reconstruction, transplantation, and even oncology. Skills and techniques from adult urology can be transferred to pediatric urology and vice versa.

In June 2015, I came across an advertisement in the Canadian Urological Association Journal for a pediatric urologist in Victoria, BC. At that time, I was a fellow at the Hospital for Sick Children and had little knowledge of how to establish a pediatric urology program. For anyone starting practice, it always helps to have a mentor who had been through the same experience him/herself. I was very fortunate to know and work with Dr. Papanikolaou. He was a great resource, providing guidance and practical advice. I also have to thank my colleagues in the division of urology and departments of pediatrics and surgery for their vision and for making this position a reality.

Establishing a pediatric urology program first starts with the needs of patients. Although based in Victoria, BC, the position included outpatient pediatric clinics across Vancouver Island. The entire island is home to 759366 people. The occasional patient may also come from mainland BC. Previously, pediatric cases were performed by adult urology colleagues or transferred to BC Children's Hospital (BCCH) in Vancouver. It was decided long before the position was posted that the island could support a pediatric urologist based on the number of cases performed on the island and transferred to $\mathrm{BCCH}$. Additionally, Victoria seemed to be experiencing an influx of young families, further adding to this need. When I was in Victoria for interviews, one person told me bluntly, "It is the land of the newly wed and nearly dead. Now, the newly weds are having kids."

Like many community urologists, I run a private clinic separate from the hospital. While this is an added cost, it also comes with greater autonomy (e.g., the ability to hire staff, select the electronic medical record system, decide on opening hours, etc.). I knew I could not afford to hire a nurse in my first year of practice, so I am more involved with the day-to-day patient care (e.g., removing catheters, postoperative checks, patient education, etc.). Conversely, my monthly clinics outside of Victoria are hospital-based and fully staffed.

With regards to diagnostic imaging, there has been reasonable access to ultrasonography, voiding cystourethrography (with experienced pediatric nurses placing the catheters),
Lasix renography, and magnetic resonance imaging. At the moment, we do not have access to dimercaptosuccinic acid (DMSA) studies and pediatric urodynamics. My colleagues at $\mathrm{BCCH}$ have been wonderful at accommodating my patients who cannot access these tests in Victoria.

My colleagues in urology and the pediatric surgical subspecialties laid the groundwork years before I started in Victoria. There is also an excellent relationship among the pediatric surgical subspecialties and we have fully autonomy over our own operating room time, separate from the adult slate. In terms of perioperative care, there is a surgical daycare, private holding area, and dedicated operating room specifically for pediatric patients. With pediatric anesthesiologists and NICU/PICU staff available, this permits surgery to be conducted safely on even premature infants and children with complex needs.

It was recognized that the pediatric urology equipment would need to be updated and added to and the administration has been very supportive of this. Where possible, I have used the existing equipment pulled from other sets (who knew that you could perform a hypospadias repair with an eye set?) or used disposable products from current contracts. I have learned how important priority-setting is and separating the needs from the wants.

\section{Conclusion}

The generalizable lessons that we've learned in the process include engaging and exciting the administration to buy-in to the importance of a program. We have learned that some individuals are supporters and some of are not supportive and one needs to find a way to appeal to both groups. It is a requisite to create interest and support from the whole team because they are an integral part of the patient experience. Furthermore, establishing priorities and defining needs and wants helps the process along. Finally, seeking opportunities outside the box will help grow centres of excellence in the community.

Competing interests: Dr. Lee has received honoraria from Pfizer. Dr. Papanikolaou reports no competing personal or financial interests.

This paper has been peer-reviewed.

Correspondence: Dr. Frank Papanikolaou, University of Toronto, The Hospital for Sick Children, and Trillium Health Partners, Toronto, ON, Canada; Frank.Papanikolaou@trilliumhealthpartners.ca 